\title{
Conceptual design of a scattering/diffraction beamline for the Russian synchrotron Ultimate Source for Synchrotron Radiation
}

\author{
M. Nentwich ${ }^{1}$, D. Novikov', O. Seeck ${ }^{1}$, T. V. Kulevoy ${ }^{2}$, M. M. Borisov' ${ }^{2}$, I. A. Subbotin ${ }^{2}$ and R. A. Senin ${ }^{2}$ \\ ${ }^{1}$ DESY Photon Science, Deutsches Elektronen-Synchrotron DESY, 22607 Hamburg, Germany \\ ${ }^{2} N R C$ "Kurchatov Institute" - Institute for Theoretical and Experimental Physics (ITEP), Moscow, 117218 Russia \\ Melanie.Nentwich@desy.de
}

The arrival of the $4^{\text {th }}$ Generation Synchrotron Rings (4GSR) facilitates a crucial step forward in the application range of modern X-ray methods. The 4GSR sources pave a way for time resolved experiments at nanometer and nanosecond resolution level and beyond. Their development therefore allows for moving the established scattering, diffraction and spectroscopy methods to the nanoscale, to combine them with microscopy at mesoscopic levels and to investigate dynamics at nanosecond time scales. The Russian national flagship Ultimate Source for Synchrotron Radiation (USSR) facility will be one of the world leading synchrotrons once it starts operation.

A major part of the existing 4GSR beamlines is dedicated to nanobeam experiments. The intended X-ray research methods must be capable of obtaining the information of the real structure at nanometer resolution levels. The required spatial resolution on the objects can vary from several interatomic spaces in modern microelectronic devices, lithium-based batteries and catalytic materials and up to tens of micrometers e. g. in research on mechanical fails or stress propagation. Moreover, the key interest concentrates on the evolution of the objects, under external influences or in the cause of device operation. Because of this great interest in nanobeam experiments, the conceptual design of nano-diffraction beamlines is of primary interest in the preparation phase of USSR.

Here, we present an analysis of the scientific cases and developments of conceptual and technical solutions for the design of a scattering/diffraction beamline. This study includes a broad overview of recent scientific cases investigated at nowadays beamlines with similar focus. Additionally, we present an extensive comparison of Nanoprobe Beamlines at 4GSR that are already operating or under construction. From those key data, we derive a model beamline for nanoprobe experiments at USSR. This generic beamline consists of (1) a double-crystal monochromator $\mathrm{Si}(111)$ with a bandwidth of $10^{-4}$, covering an energy range of $5 \mathrm{keV}$ to $40 \mathrm{keV}$, (2) a $4 \mathrm{~m}$ long tunable undulator (see Fig. 1), (3) two different combinations of focusing elements and (4) a 4+2 circle diffractometer. The beam properties of these concepts were modelled with the $\mathrm{x}$-ray tracing software xrt at different positions of the beamline. The minimal beam size at the sample position is $220 \mathrm{~nm}$ x 70nm (FWHM), see Fig. 2.

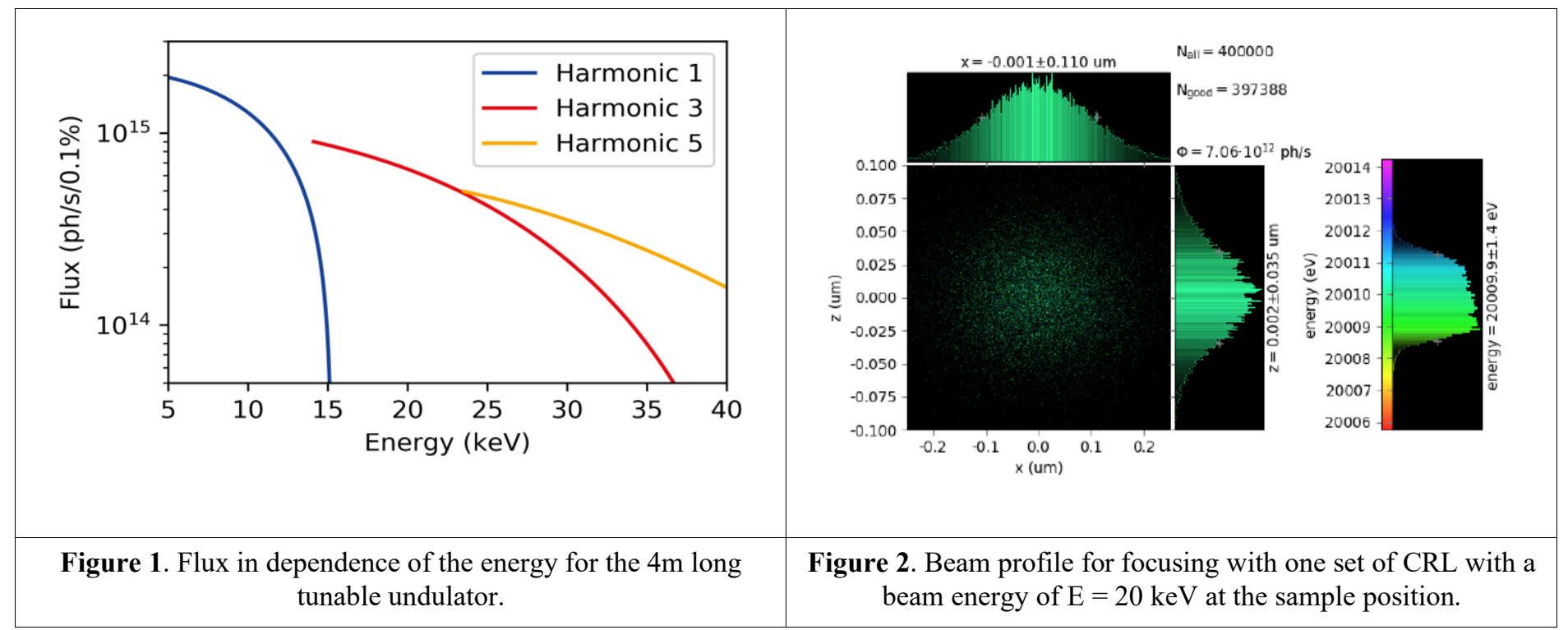

\section{Keywords: xrt; ray tracing}

Financial support within the HORIZON 2020 (H2020-EU.1.4.3.1) CREMLINplus (grant agreement ID 871072) project is cordially acknowledged. 